\title{
The prognostic value of systemic and local inflammation in patients with laryngeal squamous cell carcinoma
}

This article was published in the following Dove Press journal:

OncoTargets and Therapy

21 November 2016

Number of times this article has been viewed

\author{
Jie Wang ${ }^{1, *}$ \\ Shengzi Wang' \\ Xinmao Songl,* \\ Wenjiao Zeng ${ }^{2}$ \\ Shuyi Wang ${ }^{3}$ \\ Fu Chen' \\ Hao Ding'
}

'Department of Radiation Oncology, Eye, Ear, Nose, and Throat Hospital, Fudan University, ${ }^{2}$ Department of Pathology, Shanghai Medical School, Fudan University, ${ }^{3}$ Department of Pathology, Eye, Ear, Nose, and Throat Hospital, Fudan University, Shanghai, People's Republic of China

*These authors contributed equally to this work
Correspondence: Shengzi Wang Department of Radiation Oncology, Eye, Ear, Nose, and Throat Hospital, Fudan University, 83 Fenyang Road, Shanghai 20003I, People's Republic of China Tel +86 I89 I776 I76I

Email shengziwang@fudan.edu.cn
Background: Cancer-related systemic inflammation has been demonstrated to be associated with poor outcome in multiple types of cancers. Meanwhile, the local inflammation, which is characterized by dense intratumoral immune infiltrate, is a favorable predictor of survival outcome.

Purpose: To evaluate the role of systemic and local inflammation in predicting outcome in patients with laryngeal squamous cell carcinoma.

Patients and methods: In this retrospective study, 120 patients who had undergone postoperative radiotherapy were enrolled. Neutrophil-to-lymphocyte ratio (NLR) and plateletto-lymphocyte ratio (PLR), as calculated from pretreatment whole blood counts, were used to indicate systemic inflammation. The optimal cutoff values of NLR and PLR were determined using receiver operating characteristic curve analysis. Tumor infiltrating lymphocytes (TILs) density, as assessed by pathologist review of hematoxylin and eosin-stained slides, was used to represent local inflammation. Overall survival (OS) and recurrence-free survival (RFS) were assessed using the Kaplan-Meier method and multivariate Cox regression analysis.

Results: The best cutoff was 2.79 for NLR and 112 for PLR. Kaplan-Meier analysis revealed that high NLR, high PLR, and low TILs density were significantly correlated with inferior OS and RFS, respectively (all $P<0.05$ ). The Cox proportional multivariate hazard model showed that a high pretreatment PLR and a low TILs density were both independently correlated with poor OS and RFS, respectively (all $P<0.05$ ).

Conclusion: Markers of systemic and local inflammation, especially PLR and TILs density, are reliable prognostic factors in patients with laryngeal squamous cell carcinoma.

Keywords: laryngeal squamous cell carcinoma, systemic inflammation, neutrophilto-lymphocyte ratio, platelet-to-lymphocyte ratio, tumor infiltrating lymphocytes

\section{Introduction}

Laryngeal squamous cell carcinoma (LSCC) is one of the most common malignancies that remains as a significant cause of morbidity and mortality in head and neck. ${ }^{1}$ It is estimated that the new cases of laryngeal cancer expected among men and women in China in 2015 are 23,700 and 2,600, respectively; and the estimated number of deaths from laryngeal cancer is about $14,500 .^{2}$ Despite improvements in therapeutic modalities, the American Cancer Society indicates that there is a trend toward a decreasing 5 -year survival rate among patients with laryngeal cancer in the recent years. ${ }^{3}$ Thus, identification of novel and effective biomarkers for prediction of LSCC patients with poor prognosis or at high risk of early recurrence would be of great significance and may be beneficial for the development of effective therapeutic schemes. 
Inflammation has been regarded as a hallmark of carcinogenesis and affects many aspects of malignancy, including initiation, development, and metastasis. ${ }^{4}$ It is reported that LSCC patients have a significant increase of inflammatory response compared with chronic hypertrophic laryngitis and normal controls. ${ }^{5}$ It is known that exposure to tobacco smoke and alcohol are detrimental factors for LSCC. Mucosal damage from chronic tobacco and alcohol exposure has been well characterized. Toxins in cigarette smoke and alcohol metabolites can impair innate defenses against pathogens, modulate antigen presentation, and induce chronic inflammation at mucosal surfaces; they may also cause mutations and malignant transformation through binding to the DNA of mucosal cells. ${ }^{6,7}$ The infiltration of immune cells in LSCC, such as mast cells, neutrophils, and macrophage, may produce small molecules including cytokines, chemokines, and growth factors which can promote carcinogenesis and tumor angiogenesis and enable tumors to evade the host immune response as well as recruit more immune cells. ${ }^{8}$ What's more, squamous cell carcinomas themselves can also overexpress cytokines with pro-inflammatory, proangiogenic, and immunoregulatory activity. ${ }^{5,9}$ Additionally, studies showed that there might be genetic alterations associated with chronic inflammation in LSCC, such as the p53 mutation, and the upregulation of various inflammation-related genes, including interleukin (IL)-8, IL-6, cyclooxygenase-2, and so on. ${ }^{10,11}$

Cumulating evidence suggests that increased systemic inflammation might represent a stage-independent unfavorable prognostic factor in different types of cancer. ${ }^{1,12-15}$ Neutrophilto-lymphocyte ratio (NLR) and platelet-to-lymphocyte ratio (PLR), which reflect the systemic inflammation, are readily available and effective predictors of poor survival outcome across various cancers, including intrahepatic cholangiocarcinoma, ${ }^{12}$ breast cancer, ${ }^{13}$ colorectal cancer, ${ }^{14}$ LSCC, ${ }^{1,15}$ and so on. In contrast, a high density of tumor infiltrating lymphocytes (TILs), which represents a robust intratumoral inflammatory response, is recognized as a good prognostic marker in multiple malignancies, such as breast cancer, ${ }^{16}$ colon cancer, ${ }^{17}$ and gastric cancer. ${ }^{18}$ Recent studies have also demonstrated that high levels of intratumoral immune cell infiltrates in head and neck squamous cell carcinomas are associated with superior patient survival. ${ }^{19-22}$

So far, the present studies about LSCC have evaluated either the local or systemic inflammatory responses for their predictive abilities independently. Combination of both the systemic and local inflammation may provide more exact information to clinicians about the prognosis of LSCC. To the best of our knowledge, this is the first study to examine both the types of inflammatory processes and outcomes in LSCC with postoperative radiotherapy. Here, we examine both systemic and local inflammation, clinicopathologic data, and outcomes in a series of LSCC patients.

\section{Methods}

\section{Patients}

A retrospective study was conducted using a primary cohort of patients with LSCC who underwent surgical resection at the Department of Otorhinolaryngology, Eye, Ear, Nose, and Throat Hospital of Fudan University from January 2008 to August 2012, and then had postoperative radiotherapy or chemoradiotherapy at the Department of Radiation Oncology, Eye, Ear, Nose, and Throat Hospital of Fudan University as there were adverse features (eg, extracapsular nodal spread, positive margins, pT4 primary, N2 or N3 nodal disease, vascular embolism, and perineural invasion). Inclusion criteria were the following: histopathologically proven LSCC; complete clinical, laboratory, imaging, follow-up data, and available pathologic slides; no history of anticancer therapy; patients were treated with curative intent; no history of other malignancies, and no distant metastasis. Data relating to diagnosis, histopathological features, patient characteristics, treatment, and outcomes were collected, as well as the follow-up data, including any cancer recurrence. Approval for this study was provided by the relevant Human Research Ethics Committee of the Eye, Ear, Nose, and Throat Hospital, Fudan University. The ethics committee of the Eye, Ear, Nose, and Throat Hospital, Fudan University, did not require written informed consent be obtained from all patients, as this was a retrospective study, and all data was anonymous.

\section{Assessment of intratumoral inflammation}

Hematoxylin and eosin-stained tissue sections of formalinfixed paraffin-embedded tumor specimens were collected for all patients. One pathologist evaluated all available sections for every patient and selected the slide with the highest TILs density. ${ }^{17}$ From this slide, two certified pathologists (WJZ and SYW) scored the average TILs density within tumor areas that were composed of $>60 \%$ of neoplastic cells. ${ }^{17}$ Areas of adenoma, ulceration, and necrosis were excluded from the analysis. ${ }^{17}$ TILs density was calculated as the ratio of the area occupied by mononuclear cell infiltrates to the entire stromal area $(\% \mathrm{TIL}=$ area occupied by mononuclear cells in tumor stromal/total stromal area). ${ }^{16}$ TILs density was assessed at $\times 100$ and $\times 400$ magnification, and scored as $1+$ (low, $<30 \%$ ), $2+$ (moderate, $\geq 30 \%$ and $<60 \%$ ), or $3+$ (abundant, $\geq 60 \%$ ) by each observer. ${ }^{20}$ Pathologists were blinded from each other's assessment and patient outcomes. All the patients were assigned an overall TILs density of "high" (2+ 
or $3+$ ) or "low" (1+) by the two pathologists, ${ }^{17}$ and consensus was reached when there was a discrepancy.

\section{Assessment of systemic inflammation}

Preoperative neutrophil, platelet, and lymphocyte counts were obtained from whole blood count samples taken within 2 weeks prior to primary tumor surgery for each patient. NLR was calculated as the absolute neutrophil count divided by the absolute lymphocyte count, whereas PLR was calculated as the absolute platelet count divided by the absolute lymphocyte count.

\section{Statistical methods}

Statistical analyses were performed using IBM SPSS version 19.0 (IBM, Armonk, NY, USA) and GraphPad VR Prism 6 (GraphPad Software Inc., La Jolla, CA, USA). Overall survival (OS) was defined as time from the date of diagnosis to death from any cause. Relapse-free survival (RFS) was calculated as time to relapse of LSCC, excluding death events or diagnosis of a second malignancy. Survival curves for OS and RFS were analyzed using the Kaplan-Meier method and compared using the log-rank test. The best cutoffs for NLR and PLR were determined using a time-dependent receiver operating characteristic curve. Proportions were compared using the $\chi^{2}$ method and Fisher's exact test. Multivariate analyses of survival were performed using the Cox proportional hazards model; data were presented as hazard ratios (HRs) and 95\% confidence intervals (CIs). Two-tailed $P$-values $<0.05$ were considered to be statistically significant.

\section{Results}

\section{Patients}

A total of 120 patients were eligible for the study. The mean age at diagnosis was $60.6 \pm 8.6$ years. There were 118 men $(98.3 \%)$ and 2 women $(1.7 \%)$. The majority of patients were current or ex-smokers ( $\mathrm{n}=90,75.0 \%)$, and $66(55.0 \%)$ had a history of alcohol intake. The site of the primary tumor was almost distributed between the glottis (63 [52.5\%]) and supraglottic larynx (52 [43.3\%]), and only five persons (4.2\%) had subglottic cancer. Sixty patients $(50.0 \%)$ were in T1-T2 stage and the others $(50.0 \%)$ were in T3-T4 stage. Eighty-two patients were in N0 stage (68.3\%), with 38 patients (31.7\%) in N1-N3 stage. Among the 63 patients with glottis cancer, only 12 patients were in $\mathrm{T} 1$ stage and the other 51 patients were in T2-T4 stage, which may invade the supraglottic or subglottic region, paraglottic space, and other nearby structures. All the 52 patients with supraglottic cancer were in T2-T4 stage. Among the 38 patients with cervical node metastasis, only 11 patients had glottis cancer and all the others had supraglottic cancer. Additionally, all the 11 patients with glottis cancer who also had cervical node metastasis were all in T2-T4 stage. Surgical procedures included total laryngectomy (55 cases, $45.8 \%$ ), partial laryngectomy (54 cases, $45.0 \%$ ), and $\mathrm{CO}_{2}$ laser surgery (11 cases, 9.2\%). All the patients had postoperative radiotherapy, in which 49 patients had chemotherapy as well. Patient and tumor characteristics were listed in Table 1 for more details. No significant correlations were found between NLR, PLR, and clinicopathological characteristics in our study (Table S1).

\section{Survival analyses based on markers of systemic inflammation}

In all the 120 LSCC patients, the mean survival time was 40.1 \pm 14.9 months (from 5.0 to 91.0 months), and mean RFS was $32.7 \pm 19.2$ months (from 4.0 to 64.0 months). Using time-dependent receiver operating characteristic curves, we determined a cutoff NLR value of 2.79 and a cutoff PLR value of 112 for prognosis in LSCC patients. The mean OS time was 41.1 \pm 14.9 months in the low NLR group and $38.3 \pm 14.7$ months in the high NLR group. As shown in Figure 1A, the 5-year OS rate of the patients with an NLR $\geq 2.79$ was significantly lower than that of patients with an NLR $<2.79$ (44.1\% vs $57.6 \%, P=0.020)$. We also found that an NLR $\geq 2.79$ was significantly correlated with LSCC recurrence (NLR $<2.79$ vs NLR $\geq 2.79$, mean RFS $34.7 \pm 20.0$ vs $29.0 \pm 17.2$ months). The 5 -year RFS rates in the high NLR group were significantly lower compared with the low NLR group ( $42.7 \%$ vs $65.9 \%, P=0.017$, Figure 1B).

The survival analysis also highlighted the poor outcome of the patients with an elevated PLR. A high pretreatment

Table I Patients' clinicopathological characteristics, $n=120$

\begin{tabular}{ll}
\hline Characteristics & Values \\
\hline Age (years), mean \pm SD (range) & $60.6 \pm 8.6(40-8 I)$ \\
Sex (male/female) & 1 I $8(98.3 \%) / 2(I .7 \%)$ \\
Smoking (no/yes) & $30(25.0 \%) / 90(75 \%)$ \\
Drinking (no/yes) & $54(45 \%) / 66(55 \%)$ \\
Tumor subsite (supraglottic/ & $52(43.3 \%) / 63(52.5 \%) /$ \\
glottic/subglottic) & $5(4.2 \%)$ \\
T stage (TI-T2/T3-T4) & $60(50 \%) / 60(50 \%)$ \\
$\mathrm{N}$ stage (NO/NI-N3) & $82(68.3 \%) / 38(3 I .7 \%)$ \\
TNM stages (I-II/III-IV) & $39(32.5 \%) / 8 I(67.5 \%)$ \\
Pathological type (highly/ & $24(20.0 \%) / 84(70.0 \%) /$ \\
moderately/poorly) & $12(10.0 \%)$ \\
NLR (<2.79/ $\geq 2.79)$ & $78(65.0 \%) / 42(35.0 \%)$ \\
PLR ( $<I I 2 / \geq I I 2)$ & $58(48.3 \%) / 62(5 I .7 \%)$ \\
TILs density (low/high) & $56(46.7 \%) / 64(53.3 \%)$ \\
\hline Notes: Values in parentheses are percentages unless indicated otherwise; according \\
to the 7th American Joint Committee on Cancer staging system. \\
Abbreviations: NLR, neutrophil-to-lymphocyte ratio; PLR, platelet-to-lymphocyte \\
ratio; TILs, tumor infiltrating lymphocytes.
\end{tabular}



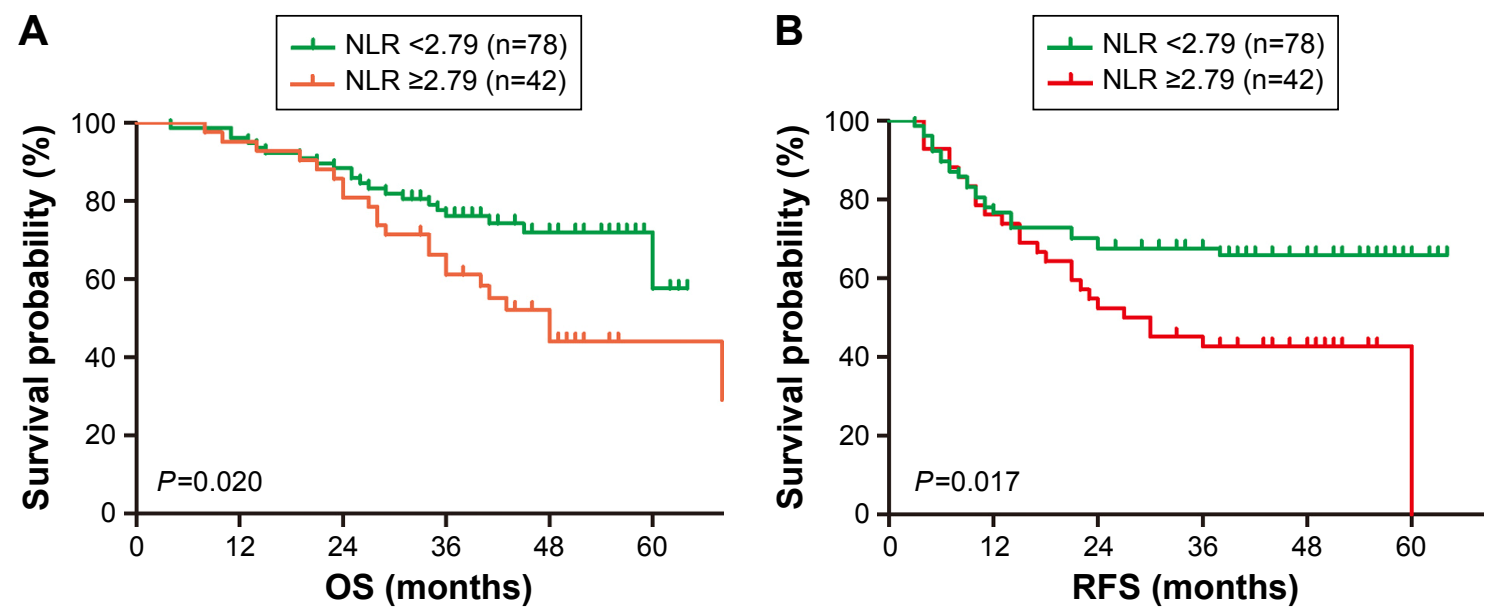

Figure I Kaplan-Meier survival curves for (A) OS and (B) RFS of I 20 LSCC patients stratified as NLR $<2.79$ vs NLR $\geq 2.79$.

Abbreviations: OS, overall survival; RFS, recurrence-free survival; LSCC, laryngeal squamous cell carcinoma; NLR, neutrophil-to-lymphocyte ratio.

PLR was found to be associated with worse OS (PLR $<112$ vs $P L R \geq 112$, mean OS $42.2 \pm 14.5$ vs $38.2 \pm 15.0$ months). The 5-year OS rates in the low PLR group were significantly higher compared with the high PLR group (77.8\% vs $28.7 \%$, $P=0.0007$, Figure 2A). Moreover, high pretreatment PLR was also associated with worse RFS (PLR $<112$ vs PLR $\geq 112$, mean RFS $37.0 \pm 18.2$ vs $28.7 \pm 19.4$ months). The 5-year RFS rate in the high PLR group (41.8\%) was significantly lower compared with the low PLR group $(62.5 \%, P=0.0007$, Figure $2 \mathrm{~B}$ ). These data suggested that high PLR may be a marker of early LSCC recurrence.

\section{Survival analyses based on marker of intratumoral inflammation}

In our study, 56 patients had low TILs $(<30 \%$, Figure $3 \mathrm{~A})$, 41 patients had moderate TILs ( $\geq 30 \%$ and $<60 \%$, Figure $3 \mathrm{~B}$ ),

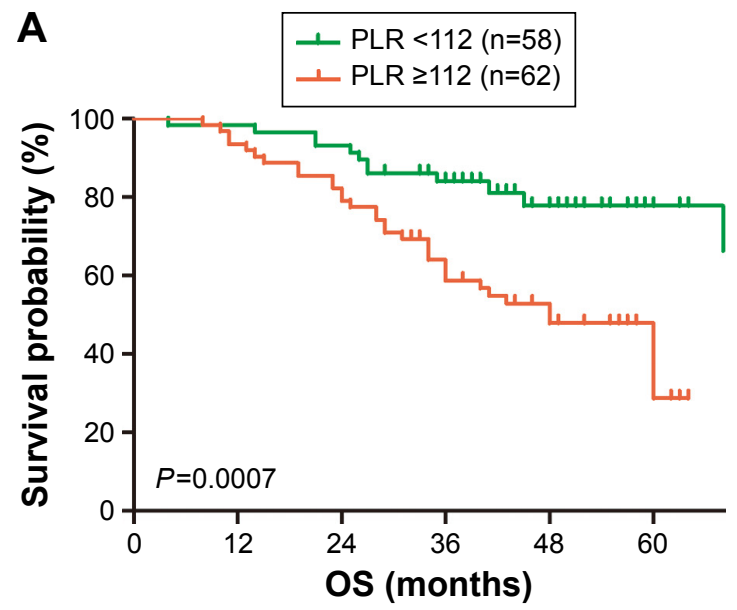

and 23 patients had abundant TILs ( $\geq 60 \%$, Figure 3C). All the patients were divided into two groups according to the TILs density: the low TILs group $(n=56)$ and the high TILs group ( $n=64)$. The mean OS time was $37.5 \pm 16.3$ months in the low TILs group and was 42.5 \pm 13.1 months in the high TILs group. The 5-year OS rates in the low TILs group $(34.0 \%)$ were significantly lower compared with the high TILs group (70.1\%, $P=0.0199$, Figure 4A). Hence, the level of stromal TILs was strongly associated with improved survival outcome of the patients. We also found that a low TILs density was significantly correlated with LSCC recurrence (low vs high TILs, mean RFS $27.3 \pm 19.0$ vs $37.5 \pm 18.2$ months). The 5-year RFS rate in the high TILs group was significantly higher compared with the low TILs group (68.5\% vs $33.1 \%, P=0.0047$, Figure 4B). The data indicated that low TILs density may predict early LSCC recurrence.

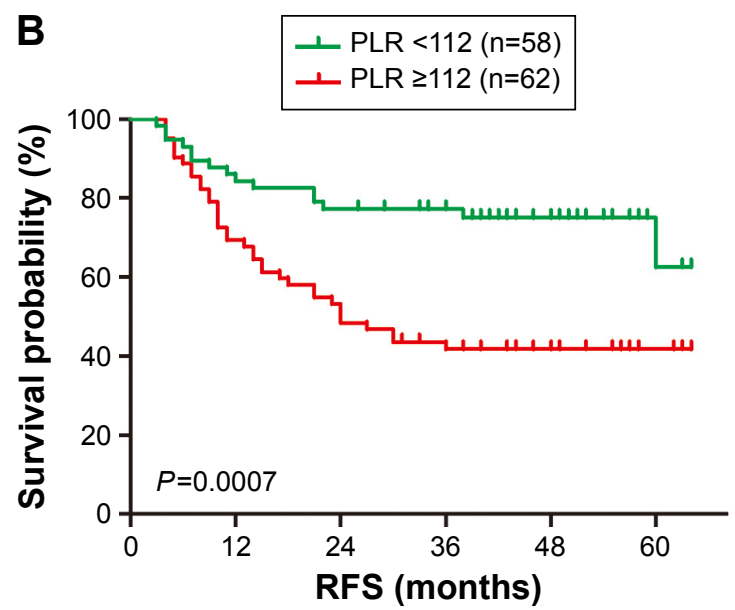

Figure 2 Kaplan-Meier survival curves for OS (A) and RFS (B) according to PLR (<II2 vs $\geq|| \mid 2)$. Notes: The PLR was significantly related to the $(\mathbf{A})$ OS $(P=0.0007)$ and $(\mathbf{B})$ RFS $(P=0.0007)$.

Abbreviations: OS, overall survival; RFS, recurrence-free survival; PLR, platelet-to-lymphocyte ratio. 

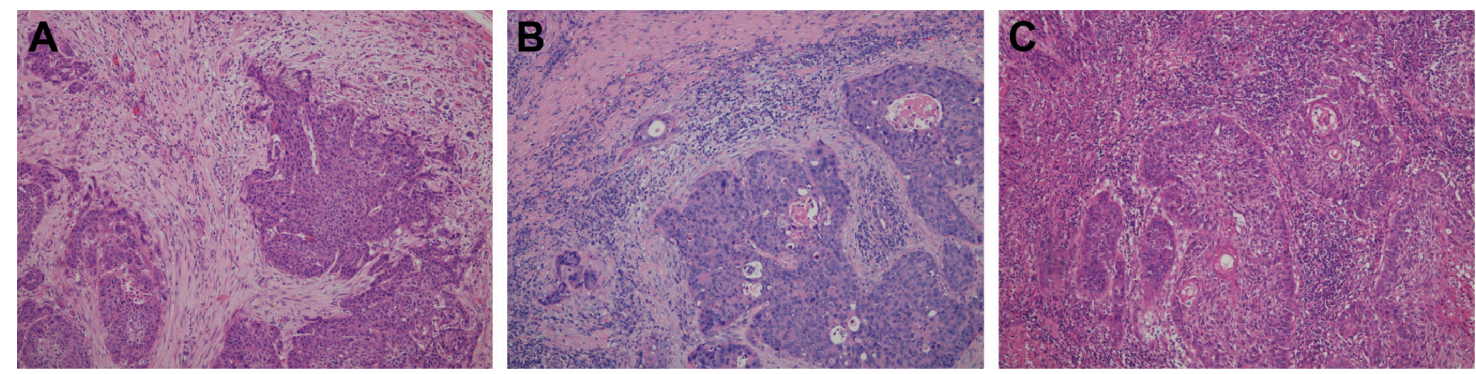

Figure 3 Stromal TILs in laryngeal squamous cell carcinomas.

Notes: (A) Low, TILs occupy an area of $<30 \%$ of the entire stromal area; (B) moderate, TILs occupy an area of $30 \%-60 \%$ of the entire stromal area; and (C) abundant, TILs occupy an area of $\geq 60 \%$ of the entire stromal area (H\&E, $\times 100$ ).

Abbreviations: TILs, tumor infiltrating lymphocytes; H\&E, hematoxylin and eosin.

What is more, no significant correlations were found between NLR and TILs, as well as between PLR and TILs $(P>0.05$, Table S1).

\section{Univariable and multivariable analyses}

Clinicopathological parameters, including NLR, PLR, and TILs density, were evaluated to identify predictors of LSCC patients' OS and RFS. The results for the statistically significant prognostic factors for OS, which were identified using univariate and multivariate analyses, were presented in Table 2. A high pretreatment NLR or PLR and a low TILs density were identified as a predictor of poor prognosis. The multivariate analyses identified that a high PLR $(P=0.004$, HR: 2.801, 95\% CI: 1.403-5.595) and a low TILs density ( $P=0.019$, HR: $0.471,95 \%$ CI: $0.251-0.884)$ were significantly correlated with worse OS (Table 2). Hence, high PLR and low TILs density were independent predictors of poor prognosis.

The statistically significant factors for RFS that were identified using univariate and multivariate analyses were presented in Table 3. A high pretreatment NLR or PLR and a low TILs density were identified as a predictor of tumor recurrence. The multivariate analyses identified high PLR ( $P=0.002$, HR: $2.622,95 \%$ CI: $1.431-4.804)$ and low TILs density ( $P=0.009$, HR: $0.473,95 \%$ CI: $0.269-0.832)$ as independent predictors of tumor recurrence (Table 3 ). These results suggested that high PLR values and low TILs density were strong predictors of tumor recurrence. Hence, LSCC patients with high PLR values and low TILs density should be closely followed for LSCC recurrence.

\section{Discussion}

Up to now, robust evidence suggests that not only the tumor behavior but also the host immune response may influence tumor outcome. ${ }^{23}$ Tumor-related systemic inflammatory response plays a crucial role in tumor growth and metastasis and is an important predictor of tumor outcome. There has been an increasing evidence that elevated NLR and PLR, which may be a simple way to assess the systemic inflammatory response, are correlated with advanced stage and poor
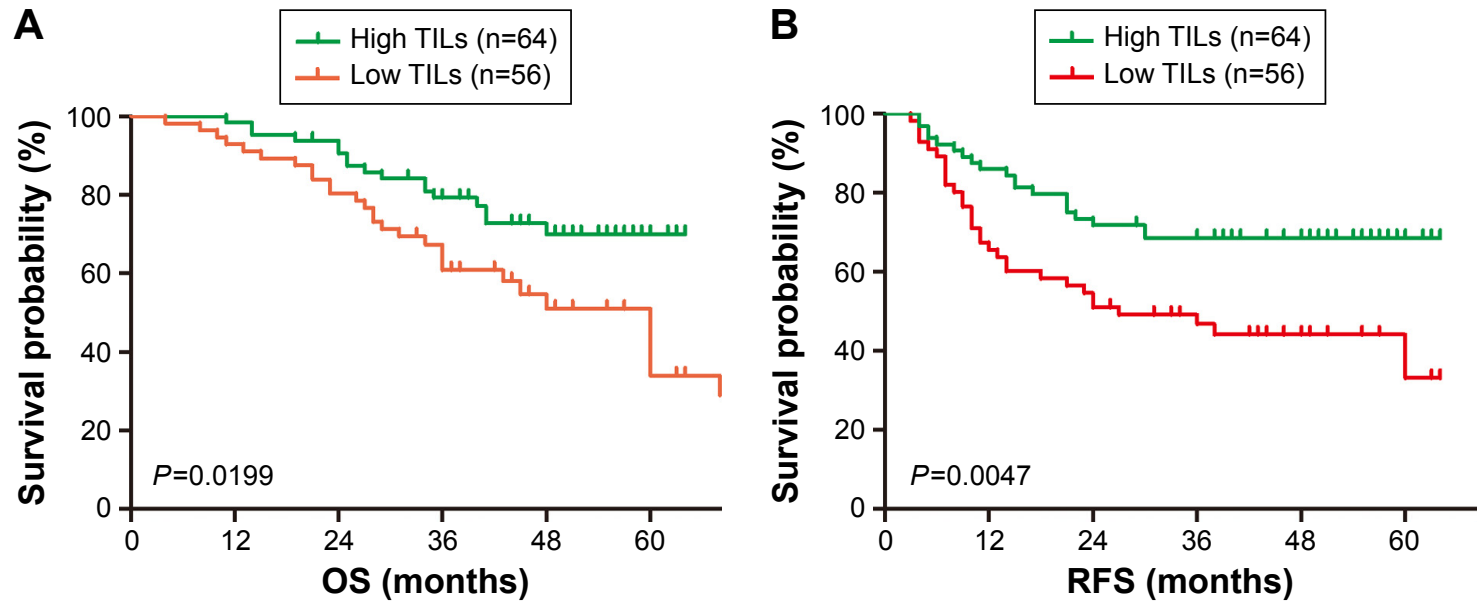

Figure 4 Kaplan-Meier curves showed that high TILs density correlated with superior OS (A) and RFS (B) of patients with LSCC, P-values calculated by log-rank test. Abbreviations: TILs, tumor infiltrating lymphocytes; LSCC, laryngeal squamous cell carcinoma; OS, overall survival; RFS, recurrence-free survival. 
Table 2 Univariate and multivariate analyses of factors in relation to overall survival using the Cox proportional hazards model $(n=120)$

\begin{tabular}{|c|c|c|}
\hline \multirow[t]{2}{*}{ Variables } & \multicolumn{2}{|l|}{ OS } \\
\hline & HR (95\% Cl) & $P$-values \\
\hline \multicolumn{3}{|l|}{ Univariate analysis } \\
\hline Age $(\leq 60$ vs $>60)$ & I.I $137(0.624-2.072)$ & 0.675 \\
\hline Smoking (no vs yes) & $0.720(0.365-1.42 I)$ & 0.344 \\
\hline Drinking (no vs yes) & $0.929(0.5 \mathrm{I} I-1.690)$ & 0.810 \\
\hline Tumor subsite (supraglottic/ & $0.601(0.179-2.020)$ & 0.436 \\
\hline glottic/subglottic) & $0.472(0.139-1.600)$ & \\
\hline $\mathrm{T}$ stage $(\mathrm{TI}+\mathrm{T} 2$ vs $\mathrm{T} 3+\mathrm{T} 4)$ & $0.885(0.486-1.613)$ & 0.690 \\
\hline $\mathrm{N}$ stage (N0 vs NI-N3) & $0.88 I(0.459-1.690)$ & 0.703 \\
\hline TNM stages $(I+I I \text { vs III + IV })^{a}$ & $1.043(0.55 \mathrm{I}-1.974)$ & 0.897 \\
\hline Pathological type (highly/ & $0.414(0.148-1.159)$ & 0.128 \\
\hline moderately/poorly) & $0.434(0.189-1.000)$ & \\
\hline NLR (low vs high) & $1.994(1.089-3.649)$ & 0.025 \\
\hline PLR (low vs high) & $3.044(1.534-6.04 I)$ & 0.001 \\
\hline TILs density (low vs high) & $0.493(0.267-0.909)$ & 0.024 \\
\hline \multicolumn{3}{|l|}{ Multivariate analysis } \\
\hline PLR (low vs high) & $2.801(1.403-5.595)$ & 0.004 \\
\hline TILs density (low vs high) & $0.47 \mathrm{I}(0.25 \mathrm{I}-0.884)$ & 0.019 \\
\hline
\end{tabular}

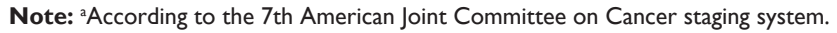
Abbreviations: NLR, neutrophil-to-lymphocyte ratio; PLR, platelet-to-lymphocyte ratio; TILs, tumor infiltrating lymphocytes; OS, overall survival; HR, hazard ratio; $\mathrm{Cl}$, confidence interval.

prognosis in patients with various solid tumors. ${ }^{1,12-15}$ Rassouli et $\mathrm{al}^{24}$ showed that pretreatment NLR and PLR could be used in head and neck squamous cell carcinomas as prognosticators of survival and recurrence independent of TNM staging. Both $\mathrm{Tu}$ et $\mathrm{al}^{1}$ and $\mathrm{Fu}$ et $\mathrm{al}^{15}$ recently demonstrated that an

Table 3 Univariate and multivariate analyses of factors in relation to recurrence-free survival using the Cox proportional hazards model $(n=120)$

\begin{tabular}{|c|c|c|}
\hline \multirow[t]{2}{*}{ Variables } & \multicolumn{2}{|l|}{ RFS } \\
\hline & HR (95\% Cl) & $P$-values \\
\hline \multicolumn{3}{|l|}{ Univariate analysis } \\
\hline Age $(\leq 60$ vs $>60)$ & $1.066(0.615-1.847)$ & 0.820 \\
\hline Smoking (no vs yes) & $0.783(0.42 \mathrm{I}-\mathrm{I} .458)$ & 0.441 \\
\hline Drinking (no vs yes) & $0.929(0.536-1.61 \mathrm{I})$ & 0.793 \\
\hline Tumor subsite (supraglottic/ & $0.712(0.214-2.375)$ & 0.640 \\
\hline glottic/subglottic) & $0.597(0.180-1.982)$ & \\
\hline $\mathrm{T}$ stage $(\mathrm{TI}+\mathrm{T} 2$ vs $\mathrm{T} 3+\mathrm{T} 4)$ & $1.127(0.650-1.955)$ & 0.670 \\
\hline $\mathrm{N}$ stage (N0 vs $\mathrm{NI}-\mathrm{N} 3)$ & $1.117(0.624-1.999)$ & 0.710 \\
\hline TNM stages $(I+I I \text { vs III + IV })^{a}$ & $1.240(0.679-2.265)$ & 0.485 \\
\hline Pathological type (highly/ & $0.656(0.232-1.853)$ & 0.721 \\
\hline moderately/poorly) & $0.743(0.3 \mid 3-1.765)$ & \\
\hline NLR (low vs high) & $1.921(1.107-3.335)$ & 0.020 \\
\hline PLR (low vs high) & $2.698(1.475-4.938)$ & 0.001 \\
\hline TILs density (low vs high) & $0.456(0.260-0.802)$ & 0.006 \\
\hline \multicolumn{3}{|l|}{ Multivariate analysis } \\
\hline PLR (low vs high) & $2.622(1.431-4.804)$ & 0.002 \\
\hline TILs density (low vs high) & $0.473(0.269-0.832)$ & 0.009 \\
\hline
\end{tabular}

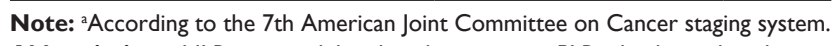
Abbreviations: NLR, neutrophil-to-lymphocyte ratio; PLR, platelet-to-lymphocyte ratio; TILs, tumor infiltrating lymphocytes; RFS, recurrence-free survival; HR, hazard ratio; $\mathrm{Cl}$, confidence interval. elevated preoperative NLR was an independent predictor of recurrence and poor OS in patients with LSCC. In our study, we also found that the pretreatment NLR had potential for use as a predictor of survival, and high NLR $(\geq 2.79)$ showed notable correlation with early recurrence and poor OS of LSCC patients. Hence, the pretreatment NLR may be a useful parameter to predict survival in LSCC patients.

The prognostic role of pretreatment PLR in patients with LSCC has, until now, been rarely investigated. We found an elevated PLR might be a significant prognostic factor in LSCC patients with both surgery and postoperative radiotherapy. The PLR values $\geq 112$ showed the greatest correlation with worse OS and early recurrence, and high PLR was independent predictor of poor prognosis. In our study, both high NLR and PLR values were associated with decreased OS (HR: 1.99, 95\% CI: $1.09-3.65, P=0.025$ and HR: 3.04, 95\% CI: 1.53-6.04, $P=0.001$, respectively) and RFS (HR: $1.92,95 \%$ CI: $1.11-3.34, P=0.020$ and HR: 2.70 , 95\% CI: $1.48-4.94, P=0.001$, respectively) in univariate analysis, but only PLR remained significant in multivariate analysis for both OS and RFS (HR: 2.80, 95\% CI: 1.40-5.60, $P=0.004$ and HR: $2.62,95 \%$ CI: $1.43-4.80, P=0.002$, respectively). These results indicated that pretreatment PLR was superior to NLR as an adverse prognostic factor in patients with LSCC, which was in accordance with Neofytou's report in colorectal cancer. ${ }^{25}$ Our results confirmed that pretreatment PLR could be reliable prognostic factors for patients with LSCC.

Although the elevated NLR and PLR appear to associate with poor RFS and OS are confirmed by many studies, the underlying mechanisms remain poorly understood. One possible explanation is that the cancer-related systemic inflammatory responses are associated with alterations in circulating blood cell distribution with a relative neutrophilia and thrombocytosis, as well as a relative depletion of lymphocytes. ${ }^{24,26}$ The increased neutrophils may produce and secrete angiogenesis-regulating growth factors, cytokines, chemokines, and proteases that could promote tumor growth and progression. ${ }^{27-30}$ Moreover, as lymphocytes have a crucial role in tumor immune surveillance as well as inhibition of tumor cell proliferation and metastasis, lymphocytopenia is assumed to reflect a generally depressed state of the host immune system..$^{24,26,31}$ It has been reported that lymphocytes in patients with head and neck cancer have significantly higher rates of apoptosis compared with normal controls, ${ }^{32}$ and the cytolytic activity of lymphocytes may be suppressed by neutrophilia. ${ }^{33}$ Platelets have been shown to have an important role in angiogenesis, tumor cell growth, and dissemination. ${ }^{34}$ The differentiation of megakaryocytes to platelets could be 
triggered by the tumor-associated inflammatory mediators, such as IL-1, IL-3, and IL-6. ${ }^{35}$ The elevated peripheral blood platelets then could accelerate tumor cell growth and dissemination through promotion of angiogenesis, increase of microvessel permeability, production of growth factors and adhesion molecules, as well as protection of the circulating cancer cells from the immune system. ${ }^{34-37}$ Hence, high NLR and PLR values may indicate an impaired host antitumor immune status. Overall, cancer-related systemic inflammation with an elevated high NLR and PLR values may potentially create a tumor-favorable microenvironment that may promote the tumor proliferation and metastasis.

Tumor infiltration by chronic inflammatory cells comprises lymphocytes, plasma cells, and macrophages. ${ }^{17}$ TILs are the major type of infiltrating immune cells and are represented by T cells, B cells, and natural killer cells. ${ }^{18}$ TILs are considered a manifestation of the host immune response against tumor cells, and several studies have already reported the potential of TILs as prognostic parameters in various human malignancies. ${ }^{18,20,38,39}$ So far, there are several methods to assess tumor immune response through lymphocytic infiltrations on formalin-fixed paraffin-embedded sections, such as morphologic evaluation of TILs, ${ }^{20}$ immunoscoring of T-cell subpopulations (eg, $\mathrm{CD}^{+}$or $\left.\mathrm{CD}^{+}\right),{ }^{40}$ and immunophenotyping. ${ }^{41}$ In our study, only the morphologic evaluation of TILs is performed. It is demonstrated that a high density of TILs is recognized as a good prognostic marker in multiple malignancies. ${ }^{16,18,20,39}$ Thus, the morphologic evaluation of TILs, which is simple and could be assessed in routine clinical practice without extra cost, may help the doctors to quickly identify patients at high risk of recurrence and early death.

The favorable prognostic role of abundant TILs has been explored in several tumors..$^{18,20,38,39}$ Vassilakopoulou et $\mathrm{al}^{20}$ reported that increased TILs density was associated with favorable outcome in LSCC. In our study, high TILs density was significantly associated with improved OS and RFS ( $P=0.0199$, 0.0047, respectively; Figure 4) of the patients and retained significance in multivariate analysis. Hence, assessment of TILs could be useful in the prediction of LSCC. Moreover, our results showed that NLR and PLR were not correlated with TILs density $(P>0.05$, Table S1). Turner et $\mathrm{al}^{17}$ also demonstrated that local and systemic inflammatory responses appeared to be largely independent of each other in colon cancer; although there was a trend toward an inverse relationship between local inflammation and systemic inflammation, it did not achieve statistical significance.

There are several limitations in the present study. First, this study is based on only 120 eligible patients, and these analyses need to be validated in a larger cohort of patients. Because LSCC is a male-dominated disease, ${ }^{2,3}$ it is inevitable that there is a significant sex bias in our patient cohort. Second, due to the limited number of enrolled patients, the stratified analysis on the basis of the combination of systemic and local inflammation is not done in our study. Finally, it is a retrospective study and is conducted at a single institution. Thus, our findings need to be validated in prospective analysis.

\section{Conclusion}

High NLR and PLR, biomarkers of the host systemic inflammatory response, are correlated with poor OS and RFS. Conversely, high TILs density, which represents the local inflammation, is predictive of longer OS and RFS. Our results also indicated that PLR and TILs density are significant independent prognostic factors in LSCC patients with both surgery and postoperative radiotherapy. Hence, the inexpensive and readily available biomarkers of systemic and local inflammation, especially PLR and TILs density, could be used for risk assessment in clinical practice for individual treatment and surveillance of LSCC.

\section{Acknowledgment}

The present study was supported by the Science and Technology Commission Foundation of Shanghai, China (grant number 12JC1402102).

\section{Disclosure}

The authors report no conflicts of interest in this work.

\section{References}

1. Tu XP, Qiu QH, Chen LS, et al. Preoperative neutrophil-to-lymphocyte ratio is an independent prognostic marker in patients with laryngeal squamous cell carcinoma. BMC Cancer. 2015;15:743.

2. Chen W, Zheng R, Baade PD, et al. Cancer statistics in China, 2015 CA Cancer J Clin. 2016;66:115-132.

3. Siegel R, Ma J, Zou Z, Jemal A. Cancer statistics, 2014. CA Cancer JClin. 2014;64:9-29.

4. Mantovani A. Cancer: inflaming metastasis. Nature. 2009;457:36-37.

5. Meng CD, Zhu DD, Jiang XD, et al. Overexpression of interleukin-17 in tumor-associated macrophages is correlated with the differentiation and angiogenesis of laryngeal squamous cell carcinoma. Chin Med J (Engl). 2012;125:1603-1607.

6. Zhang H, Wang Y, Bai X, et al. Cyclic tensile strain on vocal fold fibroblasts inhibits cigarette smoke-induced inflammation: implications for Reinke edema. $J$ Voice. 2015;29:13-21.

7. Peters WH, Lacko M, Te Morsche RH, Voogd AC, Oude Ophuis MB, Manni JJ. COX-2 polymorphisms and the risk for head and neck cancer in white patients. Head Neck. 2009;31:938-943.

8. Gastardelo TS, Cunha BR, Raposo LS, et al. Inflammation and cancer: role of annexin A1 and FPR2/ALX in proliferation and metastasis in human laryngeal squamous cell carcinoma. PLoS One. 2014;9:e111317.

9. Chen Z, Malhotra PS, Thomas GR, et al. Expression of proinflammatory and proangiogenic cytokines in patients with head and neck cancer. Clin Cancer Res. 1999;5:1369-1379.

10. Bonomi M, Patsias A, Posner M, Sikora A. The role of inflammation in head and neck cancer. Adv Exp Med Biol. 2014;816:107-127. 
11. Campa D, Hashibe M, Zaridze D, et al. Association of common polymorphisms in inflammatory genes with risk of developing cancers of the upper aerodigestive tract. Cancer Causes Control. 2007;18:449-455.

12. Chen Q, Dai Z, Yin D, et al. Negative impact of preoperative plateletlymphocyte ratio on outcome after hepatic resection for intrahepatic cholangiocarcinoma. Medicine (Baltimore). 2015;94:e574.

13. Krenn-Pilko S, Langsenlehner U, Thurner EM, et al. The elevated preoperative platelet-to-lymphocyte ratio predicts poor prognosis in breast cancer patients. Br J Cancer. 2014;110:2524-2530.

14. Chua W, Charles KA, Baracos VE, Clarke SJ. Neutrophil/lymphocyte ratio predicts chemotherapy outcomes in patients with advanced colorectal cancer. Br J Cancer. 2011;104:1288-1295.

15. Fu Y, Liu W, OuYang D, Yang A, Zhang Q. Preoperative neutrophilto-lymphocyte ratio predicts long-term survival in patients undergoing total laryngectomy with advanced laryngeal squamous cell carcinoma: a single-center retrospective study. Medicine (Baltimore). 2016;95:e2689.

16. Salgado R, Denkert C, Demaria S, et al. The evaluation of tumor-infiltrating lymphocytes (TILs) in breast cancer: recommendations by an International TILs Working Group 2014. Ann Oncol. 2015;26:259-271.

17. Turner N, Wong HL, Templeton A, et al. Analysis of local chronic inflammatory cell infiltrate combined with systemic inflammation improves prognostication in stage II colon cancer independent of standard clinicopathologic criteria. Int J Cancer. 2016;138:671-678.

18. Kang BW, Seo AN, Yoon S, et al. Prognostic value of tumor-infiltrating lymphocytes in Epstein-Barr virus-associated gastric cancer. Ann Oncol. 2016;27:494-501

19. Nguyen N, Bellile E, Thomas D, et al. Tumor infiltrating lymphocytes and survival in patients with head and neck squamous cell carcinoma. Head Neck. 2016;38(7):1074-1084. doi:10.1002/hed.24406.

20. Vassilakopoulou M, Avgeris M, Velcheti V, et al. Evaluation of PD-L1 expression and associated tumor-infiltrating lymphocytes in laryngeal squamous cell carcinoma. Clin Cancer Res. 2016;22:704-713.

21. Badoual C, Hans S, Merillon N, et al. PD-1-expressing tumor-infiltrating $\mathrm{T}$ cells are a favorable prognostic biomarker in HPV-associated head and neck cancer. Cancer Res. 2013;73:128-138.

22. Balermpas P, Michel Y, Wagenblast J, et al. Tumour-infiltrating lymphocytes predict response to definitive chemoradiotherapy in head and neck cancer. Br J Cancer. 2014;110:501-509.

23. Grivennikov SI, Greten FR, Karin M. Immunity, inflammation, and cancer. Cell. 2010;140:883-899.

24. Rassouli A, Saliba J, Castano R, Hier M, Zeitouni AG. Systemic inflammatory markers as independent prognosticators of head and neck squamous cell carcinoma. Head Neck. 2015;37:103-110.

25. Neofytou K, Smyth EC, Giakoustidis A, Khan AZ, Cunningham D, Mudan S. Elevated platelet to lymphocyte ratio predicts poor prognosis after hepatectomy for liver-only colorectal metastases, and it is superior to neutrophil to lymphocyte ratio as an adverse prognostic factor. Med Oncol. 2014;31:239.
26. Chen Q, Yang LX, Li XD, et al. The elevated preoperative neutrophilto-lymphocyte ratio predicts poor prognosis in intrahepatic cholangiocarcinoma patients undergoing hepatectomy. Tumour Biol. 2015;36: 5283-5289.

27. Fondevila C, Metges JP, Fuster J, et al. p53 and VEGF expression are independent predictors of tumour recurrence and survival following curative resection of gastric cancer. Br J Cancer. 2004;90:206-215.

28. Schaider H, Oka M, Bogenrieder T, et al. Differential response of primary and metastatic melanomas to neutrophils attracted by IL-8. Int J Cancer. 2003; 103:335-343.

29. Liu S, Li N, Yu X, et al. Expression of intercellular adhesion molecule 1 by hepatocellular carcinoma stem cells and circulating tumor cells. Gastroenterology. 2013;144:1031-1041.

30. Sivaramakrishnan V, Niranjali Devaraj S. Morin regulates the expression of NF-кB-p65, COX-2 and matrix metalloproteinases in diethylnitrosamine induced rat hepatocellular carcinoma. Chem Biol Interact. 2009; 180:353-359.

31. Coussens LM, Werb Z. Inflammation and cancer. Nature. 2002;420: 860-867.

32. Hoffmann TK, Dworacki G, Tsukihiro T, et al. Spontaneous apoptosis of circulating $\mathrm{T}$ lymphocytes in patients with head and neck cancer and its clinical importance. Clin Cancer Res. 2002;8:2553-2562.

33. el-Hag A, Clark RA. Immunosuppression by activated human neutrophils. Dependence on the myeloperoxidase system. J Immunol. 1987; 139:2406-2413

34. Sharma D, Brummel-Ziedins KE, Bouchard BA, Holmes CE. Platelets in tumor progression: a host factor that offers multiple potential targets in the treatment of cancer. $J$ Cell Physiol. 2014;229:1005-1015.

35. Alexandrakis MG, Passam FH, Moschandrea IA, et al. Levels of serum cytokines and acute phase proteins in patients with essential and cancerrelated thrombocytosis. Am J Clin Oncol. 2003;26:135-140.

36. Nierodzik ML, Karpatkin S. Thrombin induces tumor growth, metastasis, and angiogenesis: evidence for a thrombin-regulated dormant tumor phenotype. Cancer Cell. 2006;10:355-362.

37. Bambace NM, Holmes CE. The platelet contribution to cancer progression. J Thromb Haemost. 2011;9:237-249

38. Baldan V, Griffiths R, Hawkins RE, Gilham DE. Efficient and reproducible generation of tumour-infiltrating lymphocytes for renal cell carcinoma. Br J Cancer. 2015;112:1510-1518.

39. Fortes C, Mastroeni S, Mannooranparampil TJ, et al. Tumor-infiltrating lymphocytes predict cutaneous melanoma survival. Melanoma Res. 2015;25:306-311.

40. West NR, Milne K, Truong PT, Macpherson N, Nelson BH, Watson PH. Tumor-infiltrating lymphocytes predict response to anthracycline-based chemotherapy in estrogen receptor-negative breast cancer. Breast Cancer Res. 2011;13:R126.

41. Galon J, Mlecnik B, Bindea G, et al. Towards the introduction of the 'Immunoscore' in the classification of malignant tumours. J Pathol. 2014; 232:199-209 


\section{Supplementary material}

Table SI Correlation between NLR, PLR, and clinicopathological characteristics in LSCC $(n=120)$

\begin{tabular}{|c|c|c|c|c|c|c|}
\hline \multirow{2}{*}{$\begin{array}{l}\text { Clinicopathological } \\
\text { indexes }\end{array}$} & \multicolumn{2}{|l|}{ NLR } & \multirow[t]{2}{*}{$P$-values } & \multicolumn{2}{|l|}{ PLR } & \multirow[t]{2}{*}{$P$-values } \\
\hline & $<2.79(n=78)$ & $\geq 2.79(n=42)$ & & $<I 12(n=58)$ & $\geq 112(n=62)$ & \\
\hline Age (years) & & & 0.121 & & & 0.596 \\
\hline$<60$ & 45 & 18 & & 29 & 34 & \\
\hline$\geq 60$ & 33 & 24 & & 29 & 28 & \\
\hline Sex & & & 0.121 & & & 0.496 \\
\hline Male & 78 & 40 & & 58 & 60 & \\
\hline Female & 0 & 2 & & 0 & 2 & \\
\hline Smoking history & & & 0.507 & & & 0.140 \\
\hline Yes & 57 & 33 & & 47 & 43 & \\
\hline No & 21 & 9 & & 11 & 19 & \\
\hline Drinking history & & & 0.729 & & & 0.485 \\
\hline Yes & 42 & 24 & & 30 & 36 & \\
\hline No & 36 & 18 & & 28 & 26 & \\
\hline Tumor subsite & & & 0.298 & & & 0.081 \\
\hline Supraglottic & 30 & 22 & & 21 & 31 & \\
\hline Glottic & 45 & 18 & & 36 & 27 & \\
\hline Subglottic & 3 & 2 & & I & 4 & \\
\hline T stage & & & I & & & 0.144 \\
\hline $\mathrm{TI}-\mathrm{T} 2$ & 39 & 21 & & 33 & 27 & \\
\hline T3-T4 & 39 & 21 & & 25 & 35 & \\
\hline $\mathrm{N}$ stage & & & 0.593 & & & 0.804 \\
\hline No & 52 & 30 & & 39 & 43 & \\
\hline $\mathrm{NI}-\mathrm{N} 3$ & 26 & 12 & & 19 & 19 & \\
\hline TNM stages & & & 0.581 & & & 0.402 \\
\hline I-II & 24 & 15 & & 21 & 18 & \\
\hline III-IV & 54 & 27 & & 37 & 44 & \\
\hline Histological grade & & & 0.046 & & & 0.479 \\
\hline Well & 20 & 4 & & 10 & 14 & \\
\hline Moderately & 49 & 35 & & 41 & 44 & \\
\hline Poorly & 9 & 3 & & 7 & 4 & \\
\hline TILs density & & & 0.818 & & & 0.449 \\
\hline High & 41 & 23 & & 33 & 31 & \\
\hline Low & 37 & 19 & & 25 & 31 & \\
\hline
\end{tabular}

Abbreviations: NLR, neutrophil-to-lymphocyte ratio; PLR, platelet-to-lymphocyte ratio; LSCC, laryngeal squamous cell carcinoma; TILs, tumor infiltrating lymphocytes.

\section{Publish your work in this journal}

OncoTargets and Therapy is an international, peer-reviewed, open access journal focusing on the pathological basis of all cancers, potential targets for therapy and treatment protocols employed to improve the management of cancer patients. The journal also focuses on the impact of management programs and new therapeutic agents and protocols on patient perspectives such as quality of life, adherence and satisfaction. The manuscript management system is completely online and includes a very quick and fair peer-review system, which is all easy to use. Visit http://www.dovepress.com/testimonials.php to read real quotes from published authors. 